\title{
Nitrogen gas spillage in a confined space located in the Gran Sasso Underground Nuclear Physics Laboratory: an outstanding oxygen deficiency hazard case study
}

\author{
G. Bonfini, F. Gabriele, M. Tobia, R. Tartaglia \& A. Giampaoli \\ PPS Department, National Institute of Nuclear Physics, \\ Gran Sasso National Laboratory, Assergi, L'Aquila, Italy
}

\begin{abstract}
This paper deals with the cryogenic risk arising from nitrogen spillage in a box located in Hall A of the underground laboratories of the Gran Sasso National Laboratory, Assergi (L'Aquila). This box will be used as a storage area for some sensitive material (crystals) which will be used for an experiment called "CUORE". Starting from the Fault Tree Analysis (FTA), in order to estimate the global risk for human activities, a consequence analysis has been performed, using EFFECTS software models. The gap between the latest and the real scenario has been covered by a real test during which a "monitored, measured and controlled" gas nitrogen flow has been spilled into the box. Furthermore, a description of a reliable base for computational fluid dynamics (CFD) analysis is treated.
\end{abstract}

Keywords: safety, cryogenic, ODH, FTA, CFD, confined space, underground laboratory.

\section{Introduction}

Although the experiments housed in the LNGS underground site have been constructed and maintained according to the highest engineers standards level and guidelines, the strong and continuous demand of new operations and facilities needed to improve the experiment data acquisition, induces the PPS department to daily work in finding the best trade-off between scientific results and human safety. 
Since most of the experiments request the use of compressed and liquefied gases, the PPS is often involved in the study and design of new solutions to keep within the LNGS acceptable range [1] the risk of human exposure to a reducedoxygen atmosphere in a confined space.

As a part of this job, in the present paper is described the activities carried out to manage the oxygen deficiency hazard $(\mathrm{ODH})$ arising from the nitrogen spillage in a box located in Hall A of the underground laboratory.

\section{Real test and theoretical approach}

This paper describes the activities carried out by the PPS department of the Gran Sasso National Laboratory to study and manage the risk caused by gas Nitrogen release, in a $48 \mathrm{~m}^{3}$ box, needed to properly store the tellurium crystal used by the upcoming experiment named Cryogenic Underground Observatory for Rare Events (CUORE) [2].

The real test has been performed in the underground site to monitor the oxygen values in the box (see Figure 1) while a certain quantity $\left(7.5 \mathrm{~m}^{3} / \mathrm{h}\right.$ ) of nitrogen was spilled using two 50 litres gas bottles.

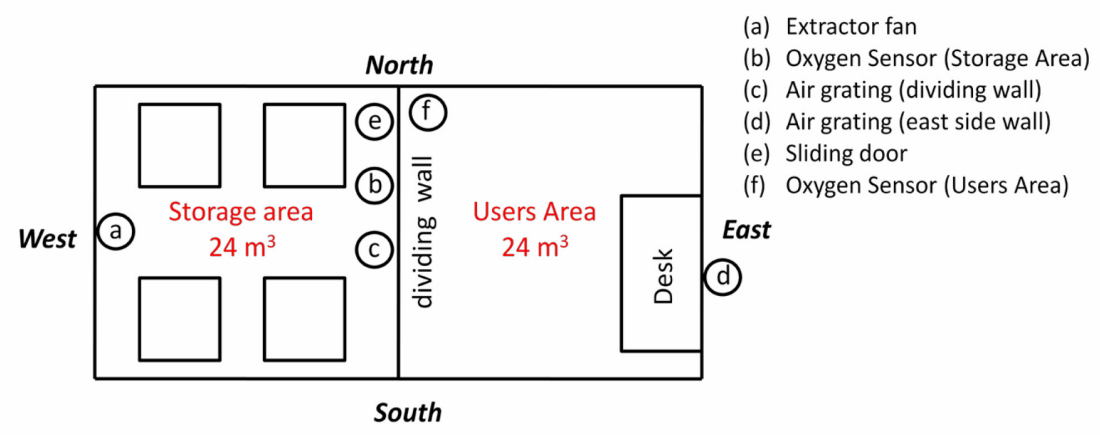

Figure 1: $\quad 48 \mathrm{~m} 3$ box diagram - view from above.

As shown in Figure 1, the box is composed of:

- a $24 \mathrm{~m}^{3}$ storage area in which the tellurium crystal will be stored inside racks (see Figure 1) and the nitrogen release will take place. A speed controlled extractor fan is located in the west side wall (see Figure 2(a));

- a $24 \mathrm{~m}^{3}$ users' area in which two PCs, a printer and a phone will be housed to allow data taking and analysis. An air grating is located in the east side wall (see Figure 2(b)) and an oxygen sensor in the north side one;

- a dividing wall in which a sliding door, an air grating and an oxygen sensor (see Figure 2(c)) are located.

With the purpose of reproducing the worst case scenario in terms of oxygen reduction rate and recovery time due to the ventilation effect, the test has been 
performed in two different phases. The first one (phase1), with the extractor fan turned off, the sliding door closed and nitrogen gas flowing at the maximum value specified by the CUORE collaboration $\left(7.5 \mathrm{~m}^{3} / \mathrm{h}\right)$. The second one (phase2), with the extractor fan operating at its minimum speed $\left(300 \mathrm{~m}^{3} / \mathrm{h}\right)$, the sliding door still closed and nitrogen still flowing at the same rate.

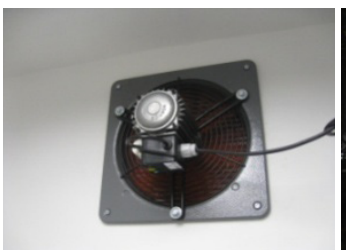

(a)

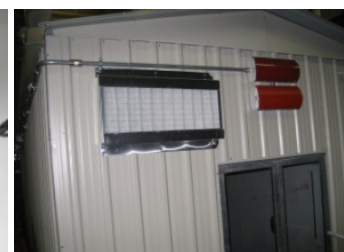

(b)

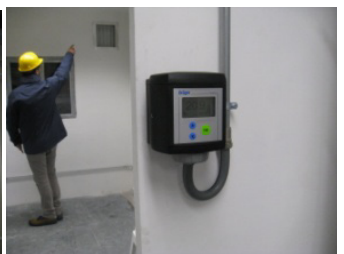

(c)

Figure 2: $\quad$ East side extractor fan, (b) west side air grating, (c) oxygen sensor.

Figure 3 shows the results obtained during phase1 and phase2, in terms of oxygen mass fraction inside the storage area.

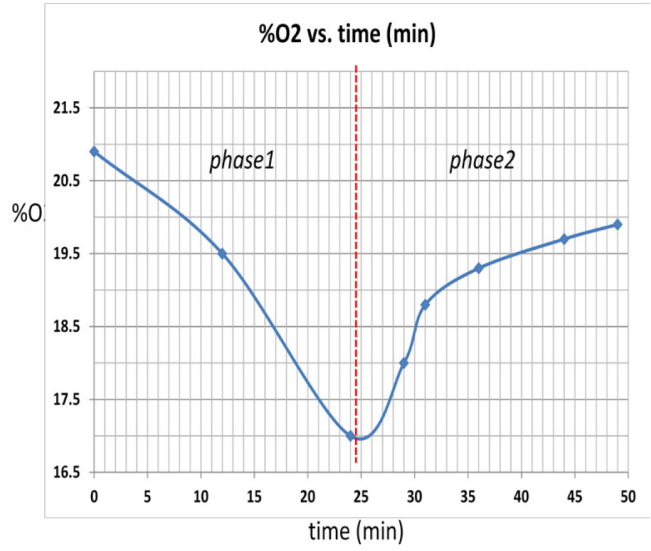

Figure 3: $\quad \% \mathrm{O}_{2}$ vs. time (min).

These results can be summarized as follows:

- phase 1: the effect of the oxygen reduction is very pronounced, as a $4 \%$ of oxygen mass fraction reduction has been measured in $24 \mathrm{~min}$;

- phase2: the recovery time for the oxygen mass fraction is quite slow, as the steady state value of $19.9 \%$ takes 25 min to be reached.

In a theoretical manner, using a discrete time approach and assuming the following conditions:

- perfect gas behaviour;

- $21 \%$ of oxygen and $79 \%$ of nitrogen represents the typical air composition; 
- negligible temperature and pressure variations;

- uniform distribution of the gas stream in the box;

- stable state reached at each time step

oxygen and nitrogen volumes $\mathrm{V}_{\mathrm{O} 2}$ and $\mathrm{V}_{\mathrm{N} 2}$ can be expressed by the following equations:

$$
\begin{aligned}
& V_{O 2}(n T)=V_{O 2}[(n-1) T]+0.21 * \text { Pext }-\frac{V_{O 2}[(n-1) T]}{V_{o}} * \text { Pext } \\
& V_{O 2}(0)=C_{0} \\
& V_{N 2}(n T)=V_{N 2}[(n-1) T]+0.79 * \text { Pext }-\frac{V_{N 2}[(n-1) T]}{V_{o}} * \text { Pext }+\delta_{N 2} \\
& V_{N 2}(0)=D_{0}
\end{aligned}
$$

where Pext is the air volume moved by extractor fan within the period of time $T$, $V_{o}$ the storage area volume $\left(\sim 24 \mathrm{~m}^{3}\right), \delta_{\mathrm{N} 2}$ the nitrogen volume released by the gas bottles within $T, \mathrm{C}_{0}$ and $\mathrm{D}_{0}$ are respectively the oxygen and nitrogen volume initial conditions. The second and third terms at the right side of the eqn (1) and eqn (2) represent the air volume introduced by the grating within time $T$ (at $21 \%$ of oxygen and $79 \%$ of nitrogen) and the one extracted by the fan.

Using the Z-transform properties, it is possible to obtain the following equation for the oxygen volume fractions during phase 1 (Pext $\sim 0, \delta_{\mathrm{N} 2} \sim 0$ ) and phase2 $\left(\beta=\operatorname{Pext} / V_{o}\right)$ :

$$
\% O_{2}(n)=\left\{\begin{array}{cc}
100 * \frac{0.21 V_{0}}{V_{o}+n * \delta_{N 2}} & \text { phase1 } \\
100 * \frac{0.21 V_{o} *\left[1-(1-\beta)^{n}\right]+(1-\beta)^{n} * C_{0}}{\left(V_{o}+\frac{\delta_{N 2}}{\beta}\right) *\left[1-(1-\beta)^{n}\right]+V_{o} *(1-\beta)^{n}} & \text { phase2 }
\end{array}\right.
$$

Figure 4 shows the comparison between the test measurements (see Figure 3 ) and the results obtained by the use of eqn(3) with $T=3 \mathrm{~min}, \delta_{\mathrm{N} 2}=0.375 \mathrm{~m}^{3}$ and $\beta=0.6$.

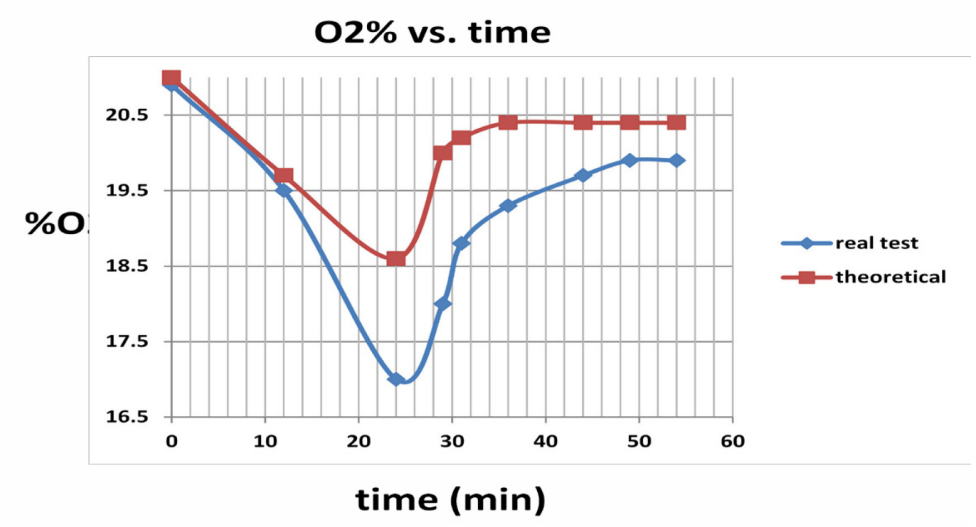

Figure 4: $\quad \mathrm{O}_{2} \%$ vs. time - real test and theoretical results. 
As shown in section 4, the non-uniform distribution of the gas stream performed during the real test can explain the difference between the theoretical and real results reported in Figure 4.

\section{Fault tree analysis}

From the results shown in the previous section, it can be concluded that the absence of ventilation in the storage area (even if temporary) can be classified as severe event, thus, as accepted by the LNGS safety standard [1], the unreliability value has to be within the range of $10^{-5}-10^{-6}$ event/year.

In order to proceed with the fault tree analysis, in the following Table1 are reported the electrical/mechanical instrumentations and human actions failure rates (or pure probability) involved in the quantitative analysis of the oxygen deficiency event in the storage area [3-5].

Table 1: Components failure rates.

\begin{tabular}{|l|c|c|c|}
\hline \multicolumn{1}{|c|}{ Component/human action } & $\begin{array}{c}\text { Failure rate } \\
\text { (event/year) }\end{array}$ & Probability & $\begin{array}{c}\text { Maintenance } \\
\text { time (months) }\end{array}$ \\
\hline n.2 UPS (redundant supply $-\mathrm{O}_{2}$ sensors) & 0.04 & & 6 \\
\hline $\mathrm{n} .1$ UPS (redundant supply - extr. fan) & 0.08 & & 6 \\
\hline Wiring & 0.26 & & 6 \\
\hline $\mathrm{n} .2 \mathrm{O}_{2}$ sensor & 0.003 & & 6 \\
\hline Extractor fan & 0.03 & & 6 \\
\hline Air gratings & & 0.003 & \\
\hline Buzzer & 0.005 & & 6 \\
\hline Human action & \multicolumn{2}{|l}{} \\
\hline
\end{tabular}

Figure 5 shows the fault tree diagram designed to be simulated by the use of Relex 7.5 software tool.

The results can be summarized as follows:

- unreliability value of about $3 * 10^{-4}$ events/year;

- the sensitivity of the tree resides in the extractor fan.

With the aim of improving the previous results, the PPS department has requested a redundant extractor fan and suggested to formalize a proper procedure to keep below 8 the amount of hours per month to be spent in the box for human activities.

The following section shows the results obtained by the use of fluid dynamics simulators as Star-CCM+ $\left(\mathrm{ADAPCO}^{\circledR}\right)$. 


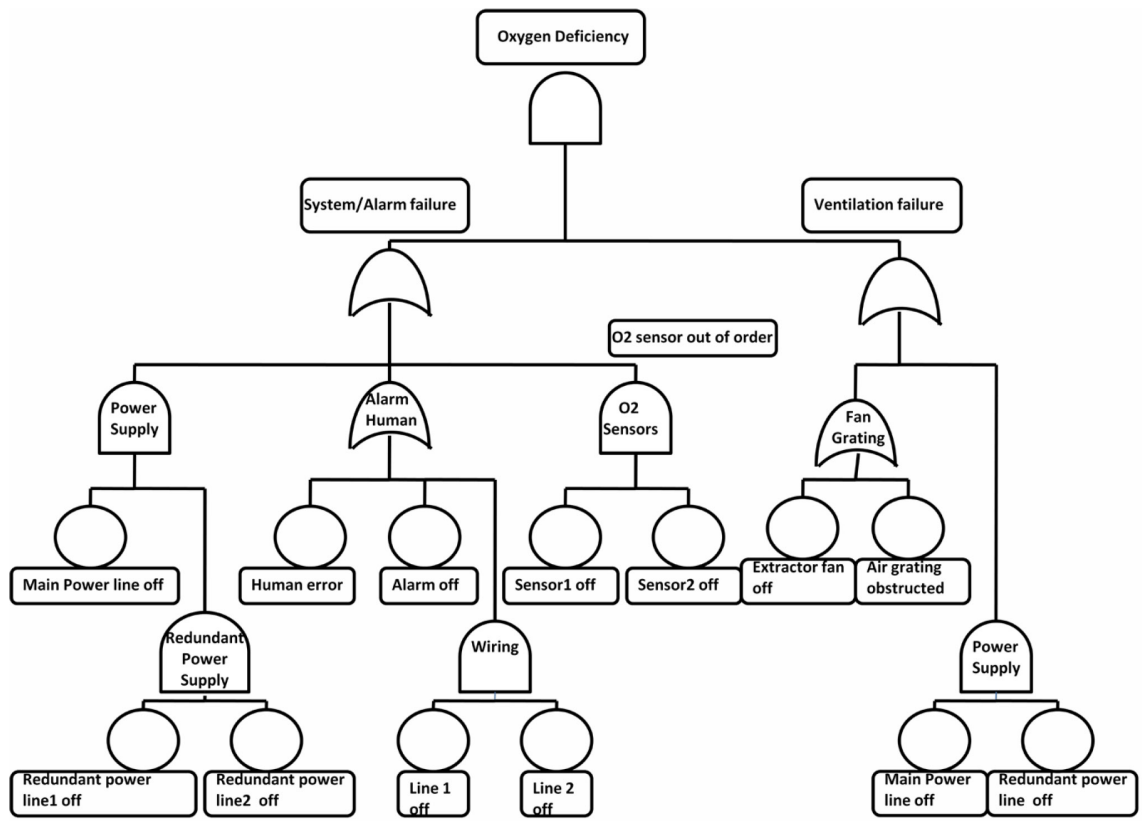

Figure 5: Fault tree diagram of the oxygen deficiency event in the storage area.

\section{CFD simulations}

In order to obtain a reliable fluid dynamic model, the real scenario has been modelled by the use of Star-CCM+ $\left(\mathrm{ADAPCO}^{\circledR}\right)$ software. The simulation results have been used to compare the model with the test scenario previously described, thus, predict the nitrogen gas effect when it will flow from the racks surfaces.

The team was initially prepared to fully investigate the cryogenic risk inside the box, then, deal with an additional test involving the instantaneous loss of liquid nitrogen from a dewar (followed by an evaporating pool) and compare the results with those from the EFFECTS $\left(\mathrm{TNO}^{\mathbb{R}}\right.$ ) simulation ("Pool Evaporation" model - Chapter 3 Yellow Book - TNO [6]).

Anyway, for safety reasons, the team decided to perform firstly the lower risk test, focusing on the gas release scenario by the use of gas bottles (for the real test) and Star-CCM+ software (for CFD simulations).

As far as the EFFECTS simulations task is concerned, the demand of optimizing the whole operation in terms of human and financial resources, drove the PPS to allocate the most of this activity within the liquid nitrogen release case study.

Returning to the Star-CCM+ simulations [7], they have been split in two phases, as in the real test described in Section 2.

Figure 6 shows the mesh model of the box that includes: 
- two gratings (external and internal) of $0.2 \mathrm{~m}$ of height and $0.6 \mathrm{~m}$ of width;

- one extractor fan in the west side wall modelled with a circle shape (radios of $0.2 \mathrm{~m})$;

- four racks in the storage area;

- two $\mathrm{N}_{2}$ sources placed in the centre of the storage area and modelled as "velocity inlet" boundary (total spillage of $7.5 \mathrm{~m}^{3} / \mathrm{h}$ );

- $\mathrm{O}_{2}$ sensor modelled as a probe and placed in the centre of the dividing wall.

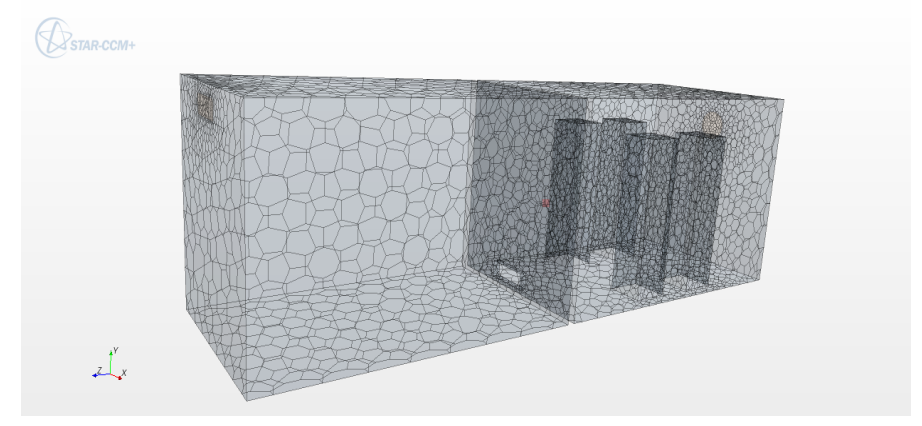

Figure 6: Mesh model of the box.

Figures 7 and 8 show, respectively, the $\mathrm{O}_{2}$ mass fraction in the storage area at the end of phasel and the velocity vectors direction and magnitude during phase2.

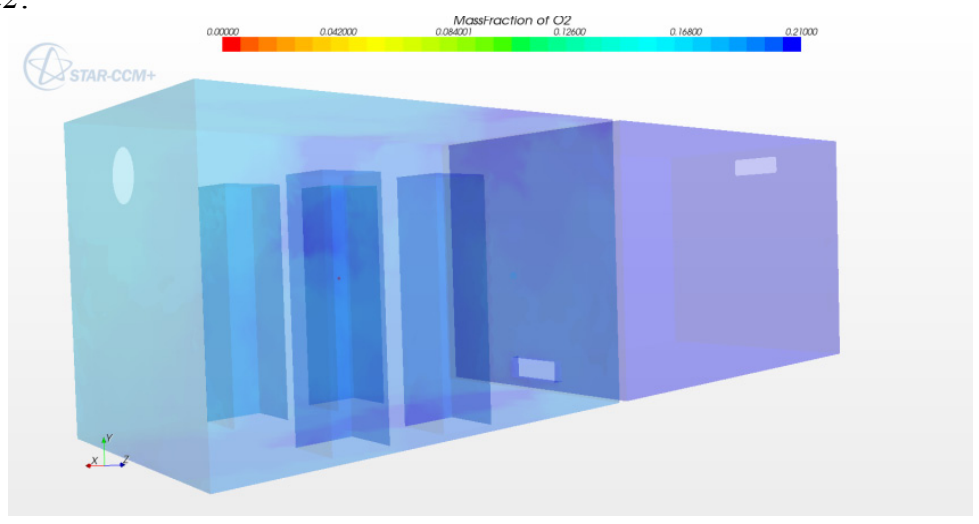

Figure 7: $\quad \mathrm{O}_{2}$ mass fraction scalar scene at the end of phase1.

It is worth nothing that the simulation results show the nitrogen gas entering the users' area during phase1, hence, the PPS department has requested the extractor fan in continuous operation. 


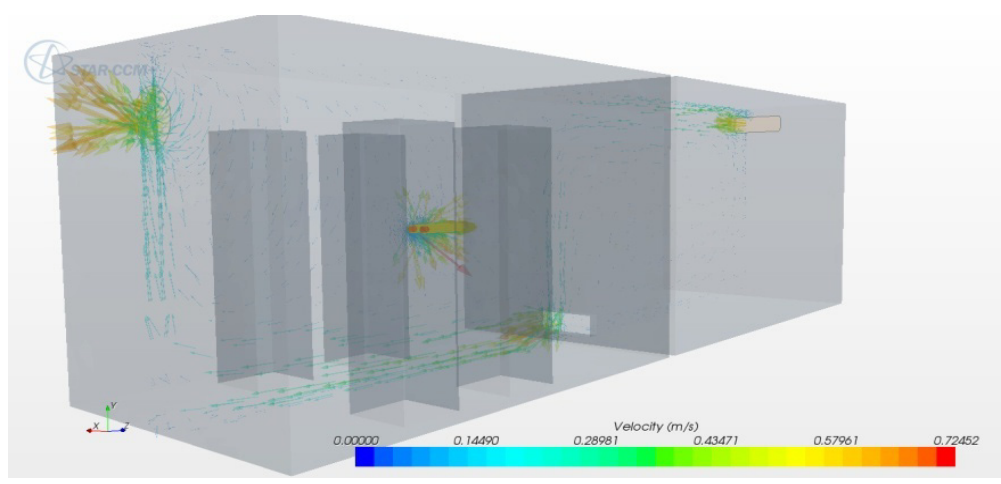

Figure 8: $\quad$ Velocity vector direction and magnitude during phase2.

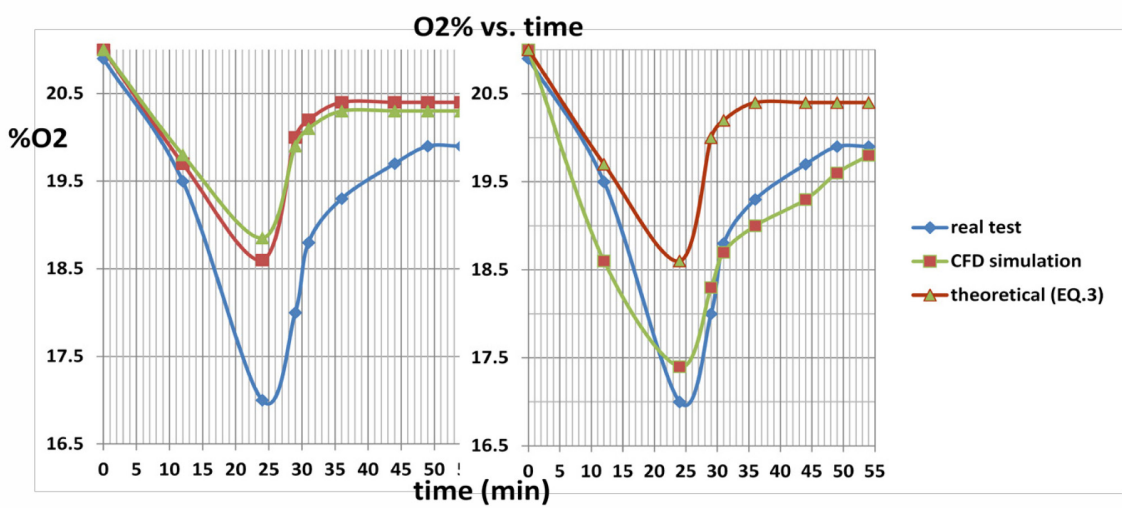

(a)

(b)

Figure 9: $\quad \mathrm{O}_{2} \%$ vs. time - comparison between real test, CFD simulation and theoretical results (a) uniform gas stream (b) non uniform gas stream.

Figure 9(a) reports a comparison among the $\mathrm{O}_{2}$ mass fraction results obtained in simulation, in test and theoretically by the eqn (3).

Figure 9(b) shows the effect of the non-uniform distribution of the gas stream inside the storage area. In this case, the simulated curve has been obtained moving the gas bottles model in proximity of the dividing wall and directing the gas streams on the $\mathrm{O}_{2}$ oxygen sensor probe (as in the real test). It is worth nothing that the new simulation scenario made the results similar to the one obtained during the real test. 


\section{Conclusions}

Prevention and Protection Service department of the Gran Sasso National Laboratory are asked to daily manage the risk inherent to the use of cryogenic fluid (as nitrogen) in a confined space.

In the LNGS underground site, where the best in class technologies are located to make possible the functionality of extremely high level scientific experiments, even a $7.5 \mathrm{~m}^{3} / \mathrm{h}$ nitrogen spillage in a closed box can represent a case to be deeply investigated in terms of safety, scientific and financial results.

In this paper we have shown how the quantitative and qualitative analysis can reveal easy solution to prevent human exposure to a reduced-oxygen atmosphere in a confined space. The measurements carried out on the real scenario and the results obtained in the FTA and CFD simulations have pointed out the presence of high risk inherent to the human activities inside the box while the nitrogen is released in the storage area. It has been demonstrated that the insertion of a redundant fan, an air grating and a simple procedure to be followed by the box users are enough to bring the risk within the LNGS strictly standards rules.

\section{References}

[1] LNGS, "Analisi Preliminare dei pericoli. Analisi e gestione dei rischi rilevanti", $5^{\text {th }}$ Revision, October 2009.

[2] Aranboldi et al. "CUORE: a cryogenic underground observatory for rare events", Nuclear Instruments and Methods in Physics Research, Section A, Accelerators, Spectrometers, Detectors and Associated, vol. 518; p. 775798, ISSN: 0168-9002.

[3] L.C. Cadwallader and S.A. Eide, "Component Failure Rate Data Sources for Probabilistic Safety and Reliability”, Process Safety Progress, vol. 29 n.3, September 2010, pp. 236-241.

[4] Frank P. Lees, "Loss in Prevention in the Process Industries", ButterworthHeinemann, April 1980, ISBN 0408106042.

[5] Fermilab ES and H Manual, "Oxygen Deficiency Hazards", November 2008.

[6] C.J.H. van den Bosh, N.J. Duijm, "Methods for the calculation of physical effects", TNO-Yellow Book, Chapter 2, Outflow and Spray release, Third Edition, 2005.

[7] CD - Adapco, "Star-CCM+ training book", Version 02/10. 